\title{
Visual pathway impairment by pituitary adenomas: quantitative diagnostics by diffusion tensor imaging
}

\author{
Ylva Lilja, MD,1,2 Oscar Gustafsson, MS, 3,4 Maria Ljungberg, PhD, 3,4 Göran Starck, PhD,,3,4 \\ Bertil Lindblom, MD, PhD, ${ }^{5}$ Thomas Skoglund, MD, PhD, ${ }^{1,6}$ Henrik Bergquist, MD, PhD, ${ }^{2,7}$ \\ Karl-Erik Jakobsson, MD, PhD, ${ }^{1,6}$ and Daniel Nilsson, MD, PhD ${ }^{1,6}$
}

\begin{abstract}
'Department of Clinical Neuroscience and Rehabilitation and ${ }^{5}$ Department of Ophthalmology, Institute of Neuroscience and Physiology, ${ }^{4}$ Department of Radiation Physics and ${ }^{7}$ Department of Otorhinolaryngology, Institute of Clinical Sciences, Sahlgrenska Academy, University of Gothenburg; and ${ }^{2}$ Ear, Nose and Throat Clinic, ${ }^{3}$ Department of Medical Physics and Biomedical Engineering, and ${ }^{6}$ Department of Neurosurgery, Sahlgrenska University Hospital, Gothenburg, Sweden
\end{abstract}

OBJECTIVE Despite ample experience in surgical treatment of pituitary adenomas, little is known about objective indices that may reveal risk of visual impairment caused by tumor growth that leads to compression of the anterior visual pathways. This study aimed to explore diffusion tensor imaging (DTI) as a means for objective assessment of injury to the anterior visual pathways caused by pituitary adenomas.

METHODS Twenty-three patients with pituitary adenomas, scheduled for transsphenoidal tumor resection, and 20 healthy control subjects were included in the study. A minimum suprasellar tumor extension of Grade 2-4, according to the SIPAP (suprasellar, infrasellar, parasellar, anterior, and posterior) scale, was required for inclusion. Neuroophthalmological examinations, conventional MRI, and DTI were completed in all subjects and were repeated 6 months after surgery. Quantitative assessment of chiasmal lift, visual field defect (VFD), and DTI parameters from the optic tracts was performed. Linear correlations, group comparisons, and prediction models were done in controls and patients.

RESULTS Both the degree of VFD and chiasmal lift were significantly correlated with the radial diffusivity $(r=0.55, p$ $<0.05$ and $r=0.48, p<0.05$, respectively) and the fractional anisotropy $(r=-0.58, p<0.05$ and $r=-0.47, p<0.05$, respectively) but not with the axial diffusivity. The axial diffusivity differed significantly between controls and patients with VFD, both before and after surgery ( $p<0.05$ ); however, no difference was found between patients with and without VFD. Based on the axial diffusivity and fractional anisotropy, a prediction model classified all patients with VFD correctly (sensitivity 1.0), 9 of 12 patients without VFD correctly (sensitivity 0.75 ), and 17 of 20 controls as controls (specificity 0.85 ).

CONCLUSIONS DTI could detect pathology and degree of injury in the anterior visual pathways that were compressed by pituitary adenomas. The correlation between radial diffusivity and visual impairment may reflect a gradual demyelination in the visual pathways caused by an increased tumor effect. The low level of axial diffusivity found in the patient group may represent early atrophy in the visual pathways, detectable on DTI but not by conventional methods. DTI may provide objective data, detect early signs of injury, and be an additional diagnostic tool for determining indication for surgery in cases of pituitary adenomas.

https://thejns.org/doi/abs/10.3171/2016.8.JNS161290

KEY WORDS pituitary adenoma; anterior visual pathways; visual impairment; diffusion tensor imaging; quantitative diagnostics, diagnostic technique; pituitary surgery

$\mathrm{P}$ ITUITARY adenomas account for $12 \%-15 \%$ of symptomatic intracranial neoplasms. ${ }^{35}$ Although their prevalence in the general population is high $-15 \%$ according to autopsy studies and $23 \%$ according to radiological studies ${ }^{11}$ - only a minority cause symptoms and re- quire treatment. The most common and earliest symptom is visual impairment, caused by tumor growth that leads to compression of the anterior visual pathways, specifically the optic chiasm. Despite ample experience of treatment by tumor resection, which leads to visual recovery in the

ABBREVIATIONS AD = axial diffusivity; DTI = diffusion tensor imaging; FA = fractional anisotropy; HRP = high-pass resolution perimetry; $M D=$ mean diffusivity; OT = optic tract; $\mathrm{RD}=$ radial diffusivity; $\mathrm{ROI}$ = region of interest; SIPAP = suprasellar, infrasellar, parasellar, anterior, and posterior; VFD = visual field defect.

SUBMITTED May 19, 2016. ACCEPTED August 8, 2016.

INCLUDE WHEN CITING Published online November 25, 2016; DOI: 10.3171/2016.8.JNS161290. 
majority of cases, ${ }^{17,25,26,31}$ there is no diagnostic tool that provides objective data about visual pathway injury to guide the treatment decision.

Standard clinical assessment of the compressive effect of pituitary adenomas is based on conventional MRI and neuroophthalmological examination. Conventional MRI will determine the size of the tumor and its relation to surrounding structures, but cannot detect functionality and possible microstructural injury of the visual pathways. Although the neuroophthalmological test battery is sensitive to visual impairment, the majority of the tests, including the visual field examination, suffer from subjectivity because the results are based on patient performance. Furthermore, the specific cause of visual impairment may be difficult to determine in the presence of another ophthalmological pathology, such as glaucoma or macular degeneration. In this study, the use of diffusion tensor imaging (DTI) as an objective tool for detection of injury of the anterior visual pathways was assessed.

DTI is an MRI technique that enables quantification of the integrity of the neural pathways, based on the amount and the directionality of water diffusion. ${ }^{2}$ The degree of directionality of diffusion, often expressed as fractional anisotropy (FA), depends primarily on the parallel organization of the axonal membranes, which has been shown to restrict diffusion. ${ }^{3,4}$ Previous studies in animal models with induced nerve injury have concluded that demyelination will lead to an increase of the diffusion perpendicular to the axons (radial diffusivity [RD]), whereas the diffusion parallel to the axons (axial diffusivity [AD]) is unaffected. Furthermore, an axonal degeneration will lead to a decrease of $\mathrm{AD}$, along with little change in $\mathrm{RD} .3,16,29$, ${ }^{30,34} \mathrm{An}$ increase in RD and/or a decrease in $\mathrm{AD}$ will result in a decrease in FA. Thus, anisotropy can be useful for detecting white matter changes, and the specific diffusivities (radial and axial) may be used to specify the type of pathology.

The aim of this exploratory study was to assess the ability of DTI to detect and grade injury in the anterior visual pathways - specifically the optic tracts (OTs) - in patients with pituitary adenomas. First, correlation between DTI parameters and neuroophthalmological outcome was assessed. Second, the ability to detect injury by DTI parameters alone was tested, by group comparisons and prediction models. The ultimate goal was to determine whether DTI can be used for objective and quantitative assessment of the anterior visual pathways in cases of pituitary adenomas, during follow-up, and in the process of determining indication for surgery.

\section{Methods}

\section{Patients and Control Subjects}

Twenty-three patients with pituitary adenomas, scheduled for transsphenoidal tumor resection, were included (age range 28-70 years, mean 52 years; 14 men) (Table 1). Inclusion criteria were age between 25 and 70 years, suprasellar tumor extension of Grades 2-4 according to the suprasellar, infrasellar, parasellar, anterior, and posterior (SIPAP) grading system, ${ }^{10}$ no other known visual impairment (apart from refractive errors), and no other known intracranial pathology, including significant trauma. The patients were included consecutively between April 2012 and August 2015 at the Sahlgrenska University Hospital. DTI, conventional MRI, and neuroophthalmological examinations were performed before and 6 months after surgery. Patients who were enrolled for and underwent surgery within 3 weeks could not be included in the study due to time constraints, because the study examinations were done separately from the clinical ones. Two patients declined to participate in the study.

Twenty healthy subjects with normal vision (apart from refractive errors) were included as controls (age range 3061 years, mean 44 years; 7 men) and underwent MRI, including DTI. All subjects except 2 underwent a standardized neuroophthalmological examination that confirmed normal vision. The remaining 2 subjects reported normal vision.

The study was approved by the regional ethical board of the University of Gothenburg and was performed according to statutes of the Declaration of Helsinki. Informed oral and written consent was obtained from all subjects prior to inclusion in the study.

\section{Neuroophthalmological Examination}

The neuroophthalmological evaluations were performed by an experienced neuroophthalmologist (B.L.), including tests for visual acuity, ocular pressure, fundus photography, and perimetry. The latter was performed using high-pass resolution perimetry (HRP) (HighTech Vision), ${ }^{14}$ which is a well-documented computerized test that is known to be comparable with conventional perimetry in cases with chiasmal syndrome. ${ }^{13}$ In HRP, also known as ring perimetry, rings of varying sizes appear on a screen in 50 different locations. The ring diameter is varied in steps of $0.1 \log _{10}$ units $(\mathrm{dB})$. In a normal visual field, there is a gradual increase in detected ring sizes from the center to the periphery. In addition to the threshold values in the individual test points, the test provides quadrant scores, that is, mean thresholds for each visual field quadrant (measured in $\mathrm{dB}$ ) (Fig. 1).

Pituitary adenomas that cause compression of the visual pathways typically first affect the upper temporal quadrant of the visual field, whereas the nasal quadrants are relatively spared. To quantify the perimetry results, the difference between the temporal and the nasal quadrant scores were calculated (temporal minus nasal), hereafter expressed as degree of visual field defect (VFD). By applying this method, each subject was made his or her own control, which was useful due to the known interindividual variability of normal perimetry results. Because VFD is the first symptom of tumor compression in most cases, the degree of VFD alone was used as a measure of visual impairment. ${ }^{12}$ For the purpose of group analysis (see below), the patient group was dichotomized into patients with and without VFD before surgery, based on the clinical evaluation by the neuroophthalmologist.

\section{MRI Protocol}

MRI was performed on a Philips Achieva 1.5T scanner. During the study, a hardware and software upgrade 


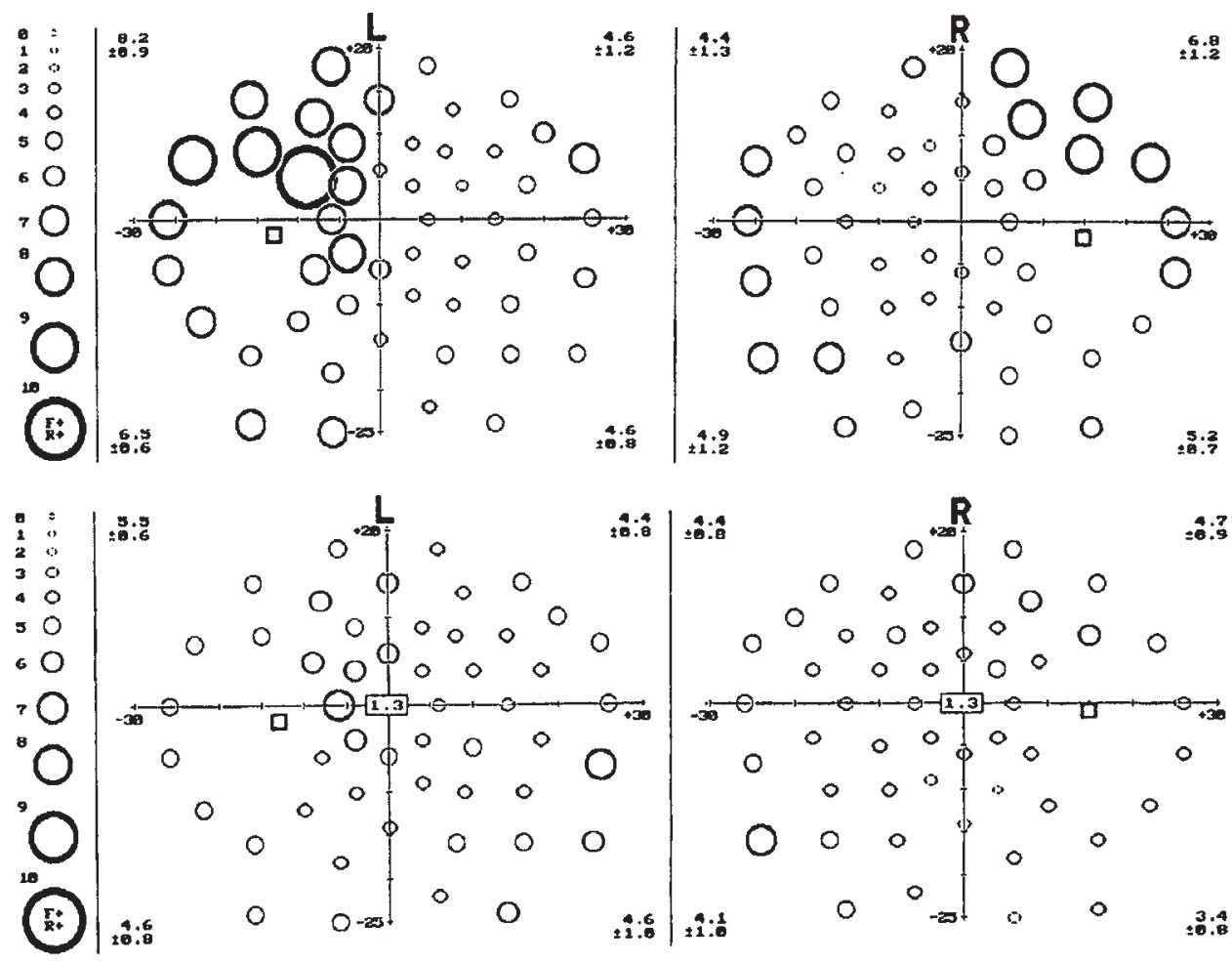

FIG. 1. Visual field examination by HRP of the left $(L)$ and right $(R)$ eyes of one of the patients. The preoperative perimetries (upper row) show typical patterns of chiasmal syndrome, with visual field impairment primarily in the upper temporal quadrants (that is, greater ring diameters). Six months after surgery (lower row), the visual fields of both eyes were normalized.

was performed, including change from analog to digital radiofrequency coils and software release change from 3.2 to 5.1. Before the upgrade, an 8-element SENSE head coil was used and after the upgrade, a 15-element SENSE head coil was used (Philips). During the scans, subjects' heads were firmly supported with cushions to reduce head motion.

A T1-weighted 3D image of the brain was acquired using a turbo field echo scan with isotropic voxel size of 1 $\mathrm{mm}^{3}$. The SENSE factor was 1.4 in the anteroposterior direction and 2 in the right-left direction.

DTI of the whole brain was performed with the following parameters: single-shot spin echo planar imaging with echo time $69 \mathrm{msec}$, axial slices, SENSE factor 3.2, half-scan factor 0.712 , isotropic $2.2 \times 2.2 \times 2.2-\mathrm{mm}$ voxels, $b=0 \mathrm{sec} / \mathrm{mm}^{2}$ (18 signals averaged) plus 32 diffusionsensitizing gradient directions $\left(b=800 \mathrm{sec} / \mathrm{mm}^{2}, 3\right.$ signals averaged), and phase encoding in the anterior-posterior direction (bandwidth $17.2 \mathrm{~Hz} / \mathrm{mm}$ ). The reconstructed inplane resolution was $1.9 \times 1.9 \mathrm{~mm}$.

All images were angulated and positioned to be parallel to the lower boundary of the corpus callosum in the sagittal view, and perpendicular to the plane of symmetry of the brain in the coronal view. The scan time was 16 minutes.

To ensure that the MR system upgrade did not affect the DTI results, 1 subject was scanned 6 times before and 6 times after the upgrade. DTI parameters from the 2 measurement occasions were compared, with focus on the OTs, resulting in no significant differences.

\section{Chiasmal Lift}

To quantify the degree of chiasmal lift by a pituitary adenoma, anatomical structures that are fairly constant in interindividual shape and location may be selected as references. Frisén and Jensen compared different such reference structures and found that the bulbopontine height correlated well with visual impairment. ${ }^{15}$ This measurement is defined as the perpendicular distance between the inferior aspect of the chiasm and the sagittal line between the dorsal-most aspect of the olfactory bulbs and the pontomesencephalic junction (Fig. 2). In the present study, the bulbopontine height was measured in the preoperative T1weighted scans of all patients.

\section{DTI Analysis}

The Oxford Centre for Functional MRI of the Brain (FMRIB) Software Library (FSL) was used for the data postprocessing (http://fsl.fmrib.ox.ac.uk/fsl/fslwiki). The voxels of the diffusion images were interpolated to an isotropic size of $1 \mathrm{~mm}^{3}$ using a sinc-like spline interpolation (FLIRT). ${ }^{19}$ Eddy current and motion correction was then performed by affine registration to the $b=0$ image..$^{19}$ The DT was calculated in each voxel within the brain using the diffusion toolbox in FSL. To characterize the anisotropy of the diffusion in each voxel, FA was calculated based on the eigenvalues of the DT.

With the goal to extract DTI parameters from the bilateral OTs in each subject, the following method was used for region of interest (ROI). The skeleton algorithm in 


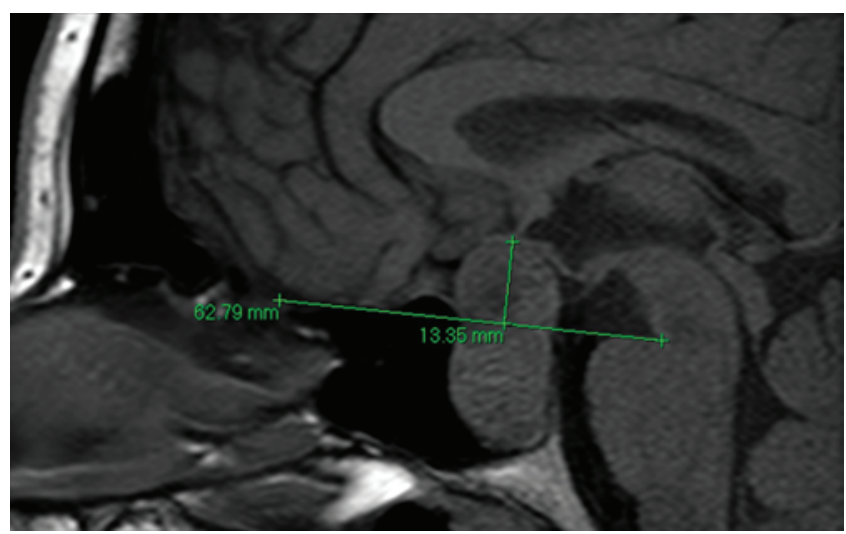

FIG. 2. Sagittal midline T1-weighted MR image of a pituitary adenoma of one of the patients. The optic chiasm (elevated and compressed by the tumor) can be seen as a light gray line above the tumor. The green lines illustrate the bulbopontine height, that is, the perpendicular distance between the inferior aspect of the chiasm and the sagittal line between the dorsal-most aspect of the olfactory bulbs and the pontomesencephalic junction. Figure is available in color online only.

tract-based spatial statistics was applied on each FA map, keeping the original space of each individual's FA map. ${ }^{28}$ Voxels with FA $<0.2$ were omitted from the resulting FA skeleton to exclude voxels that were mainly gray matter or CSF. The anterior limit for the ROI was defined as the most posterior coronal slice of the optic chiasm, which was identified by visual inspection of the color-coded FA maps. Posterior to the anterior limit, the OTs could be clearly identified in 15 continuous coronal slices in all subjects; thus, the length of an OT ROI was set to cover this $15-\mathrm{mm}$ anterior-posterior distance (Fig. 3).

A maximum of 2 voxels of the FA skeleton was selected per coronal slice. When the OT was represented by $>2$ voxels per coronal slice, 2 voxels with high FA representing the middle of the tract were selected by visual inspection. When the OT was represented by a single voxel, only this voxel was selected. When the OT was not represented by any voxels in a coronal slice, 2 voxels representing the middle of the tract were selected by visual inspection of the $b=0$ image. The latter was the case in 12 of the patients' scans ( 15 of 88 OTs), where $\geq 1$ (12 OTs) coronal slices of the OT were not included in the FA skeleton.

\section{Statistical Analysis}

For the statistical analysis, the OT ROIs were divided into 3 smaller ROIs (ROIs 1,2, and 3), because the microstructural changes (including the DTI parameters) may vary at different distances from the optic chiasm. Each ROI consisted of voxels from 5 adjacent coronal slices (Fig. 3). The 3 ROIs were analyzed separately.

\section{Correlation Analysis}

The correlation between chiasmal lift and degree of VFD, as well as chiasmal lift and the mean of the DTI parameters within each ROI, were analyzed using Pearson's correlation coefficient. A summarizing test for the association between degree of VFD and the mean of the DTI parameters within each ROI was also conducted, taking into account the fact that 2 pairs of data originated from the same subject (bilateral visual fields and OTs). Corrections for multiple comparisons were made by interpreting the individual tests as significant only if the corresponding summarizing test was significant.

\section{Group Comparisons by Mixed Model}

The subjects were divided into 5 groups to test for differences among DTI parameters in the OTs: 1) controls, 2) patients with VFD before surgery, 3) patients without

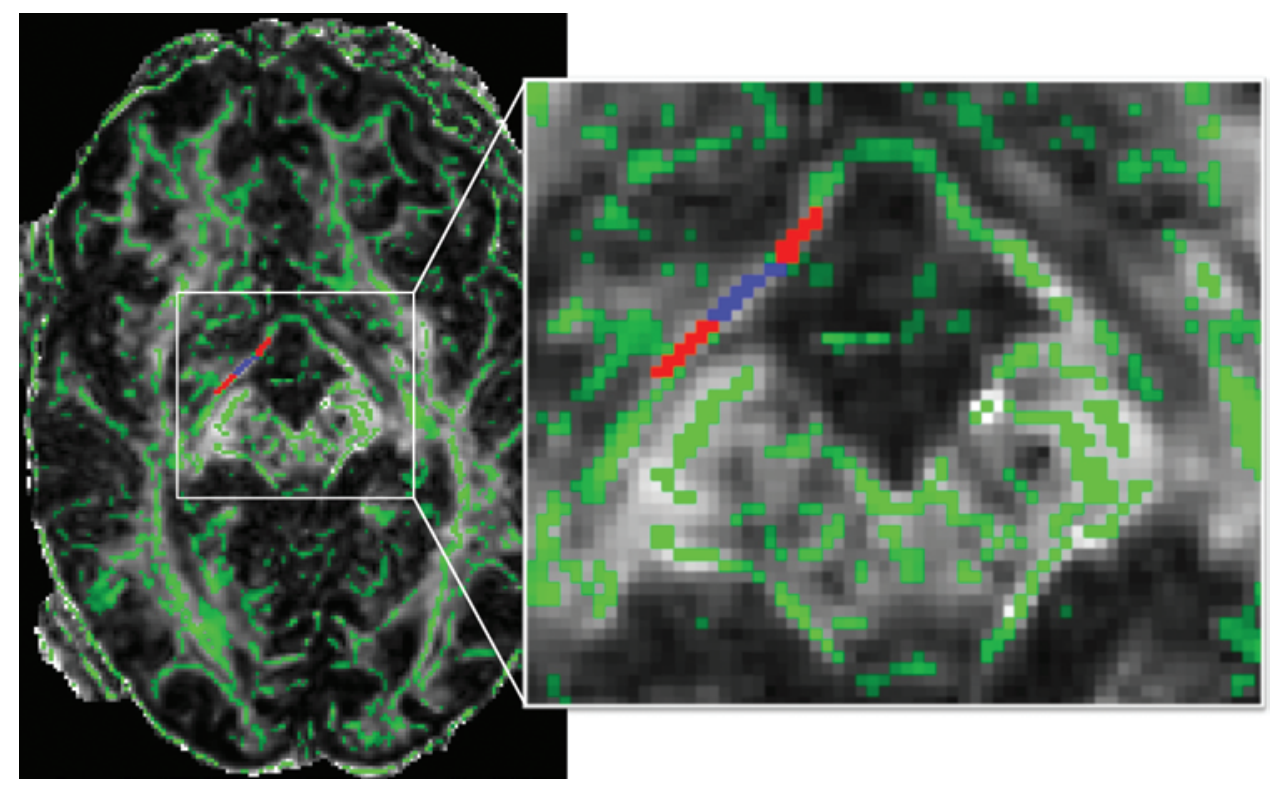

FIG. 3. Axial slice of an FA map with an overlaid FA skeleton (green voxels), including a close-up of the OT region. ROIs 1, 2, and 3 of the right OT are visualized (red and blue). Figure is available in color online only. 
VFD before surgery, 4) patients with preoperative VFD after surgery, and 5) patients without preoperative VFD after surgery. A mixed model was used for comparison of groups due to within-subject dependence, including repeated scans in the same subject (before and after surgery) and multiple data points from each subject, originating from the bilateral OTs as well as from multiple voxels within each ROI. The results were adjusted for age, assuming a linear change in outcome with age within each side and ROI. Pairwise comparisons of groups were made, adjusting confidence intervals and $\mathrm{p}$ values within each ROI for multiple comparisons using the Tukey-Kramer method.

\section{Classification by Prediction Model}

A prediction model using logistic regression was constructed to test the capability of DTI parameters to correctly classify a subject as a patient (before surgery) or as a control. The means of each of the 4 DTI parameters per ROI were included as potential predictors, resulting in 12 candidates. Forward selection was used to identify the combination of predictors with the best discriminative ability, and the model with the smallest Akaike information criterion value was selected. The procedure was performed for models using 2, 3, and 4 predictors. Models using more predictors were disregarded because they resulted in higher Akaike information criterion values and to prevent overfitting due to the limited number of subjects. Leave-one-out cross-validation was performed to validate and compare the models. The best model was selected based on cross-validation results, statistical significance of included predictors, as well as the lack of strong correlations between included predictors.

\section{Results}

\section{Preoperative and Postoperative Neuroophthalmology}

Eleven of the 23 patients had pathological visual fields before surgery. Five of the patients with VFD also had impaired visual acuity (defined as $<0.8$ ). All patients with VFD improved after surgery, although 4 patients had remaining VFD at the evaluation 6 months after surgery. Three patients showed clinical signs of optic nerve atrophy, which remained unchanged after surgery (Table 1).

\section{Statistical Analysis}

In contrast to the statistical results based on DTI parameters from ROIs 2 and 3, none of the statistical analyses had significant results when DTI parameters from ROI 1 were included. Results from ROI 1 are therefore not further presented.

\section{Correlations Among DTI Parameters, VFD, and Chiasmal Lift}

There was a significant positive correlation between degree of chiasmal lift and VFD $(r=0.72, p=0.0001)$. Furthermore, there were significant positive correlations between RD and degree of VFD $(r=0.55, \mathrm{p}<0.05)$ and chiasmal lift $(r=0.48, p<0.05)$, negative correlations between FA and degree of VFD $(r=-0.58, \mathrm{p}<0.05)$ and
TABLE 1. Patient demographic data and clinical characteristics*

\begin{tabular}{|c|c|c|c|c|c|c|}
\hline $\begin{array}{l}\text { Case } \\
\text { No. }\end{array}$ & $\begin{array}{c}\text { Age } \\
\text { (yrs), } \\
\text { Sex }\end{array}$ & $\begin{array}{l}\text { Preop } \\
\text { VFD }\end{array}$ & $\begin{array}{c}\text { Degree } \\
\text { of } \\
\text { Preop } \\
\text { VFD } \\
\text { (dB) }\end{array}$ & $\begin{array}{l}\text { Chiasm } \\
\text { Lift } \\
(\mathrm{mm})\end{array}$ & $\begin{array}{c}\text { Op } \\
\text { Indication }\end{array}$ & Comment \\
\hline 1 & $58, \mathrm{M}$ & Yes & 6.4 & 13 & VFD & \\
\hline 2 & $65, M$ & No & -0.2 & 11 & $\begin{array}{l}\text { Chiasm } \\
\text { compr }\end{array}$ & \\
\hline 3 & $66, M$ & No & 0.7 & 17 & $\begin{array}{l}\text { Chiasm } \\
\text { compr }\end{array}$ & \\
\hline 4 & $35, \mathrm{M}$ & Yes & 4.8 & 14 & VFD & \\
\hline 5 & $42, \mathrm{M}$ & No & 0.1 & 3 & Acro & \\
\hline 6 & $50, F$ & Yes & 5.3 & 13 & VFD & \\
\hline 7 & $62, \mathrm{M}$ & Yes & NA & 12 & VFD & $\begin{array}{l}\text { No HRP, small } \\
\text { postop VFD }\end{array}$ \\
\hline 8 & $46, M$ & Yes & 4.8 & 9 & VFD & $\begin{array}{r}\text { Atrophy, small } \\
\text { postop VFD }\end{array}$ \\
\hline 9 & $45, \mathrm{~F}$ & Yes & 4 & 8 & VFD & \\
\hline 10 & $45, F$ & No & -0.2 & 3 & Acro & \\
\hline 11 & $53, \mathrm{M}$ & Yes & 3.3 & 4 & VFD & \\
\hline 12 & $68, M$ & No & 1.2 & 7 & $\begin{array}{l}\text { Chiasm } \\
\text { compr }\end{array}$ & \\
\hline 13 & $66, M$ & Yes & 8.2 & 13 & VFD & $\begin{array}{l}\text { Small postop } \\
\text { VFD }\end{array}$ \\
\hline 14 & $28, F$ & Yes & 5.2 & 13 & VFD & \\
\hline 15 & $70, M$ & No & -3.2 & 6 & $\begin{array}{l}\text { Chiasm } \\
\text { compr }\end{array}$ & \\
\hline 16 & $40, F$ & No & 0.4 & 1 & Pain & \\
\hline 17 & $35, F$ & No & -0.5 & 9 & Acro & \\
\hline 18 & $60, M$ & Yes & 21.2 & 23 & VFD & $\begin{array}{l}\text { Postop VFD, } \\
\text { atrophy }\end{array}$ \\
\hline 19 & $69, M$ & No & 0.3 & 11 & $\begin{array}{l}\text { Chiasm } \\
\text { compr }\end{array}$ & \\
\hline 20 & $28, F$ & Yes & 11.8 & 18 & VFD & Atrophy \\
\hline 21 & $33, F$ & No & 1.6 & 9 & $\begin{array}{l}\text { Chiasm } \\
\text { compr }\end{array}$ & \\
\hline 22 & $67, M$ & No & -3.0 & 10 & Acro & $\begin{array}{l}\text { Discontinued } \\
\text { postop }\end{array}$ \\
\hline 23 & $67, \mathrm{~F}$ & No & 0.6 & 5 & $\begin{array}{l}\text { Chiasm } \\
\text { compr }\end{array}$ & $\begin{array}{l}\text { Discontinued } \\
\text { postop }\end{array}$ \\
\hline
\end{tabular}

Acro $=$ acromegaly; chiasm compr $=$ chiasmal compression; $\mathrm{NA}=$ not ap plicable; pain $=$ headache/facial pain.

* Indications for surgery were VFD $(n=11)$, radiological chiasmal compression $(n=7)$, acromegaly $(n=4)$, and headache/facial pain $(n=1)$. Only 1 patient (Case 18) had a postoperative VFD according to measures based on the HRP results (difference between lateral and nasal quadrants), whereas 4 patients had a postoperative VFD based on the evaluation by the neuroophthalmologist. One patient (Case 7) was not evaluated by HRP and was thus excluded from the statistical correlations. Two patients (Cases 22 and 23) discontinued the study postoperatively, due to disease (Case 22) and upon the patient's request (Case 23). Most patients had tumors of suprasellar SIPAP Grade 3; the remaining tumors (Cases 5, 10, and 16) had suprasellar SIPAP Grade 2. 

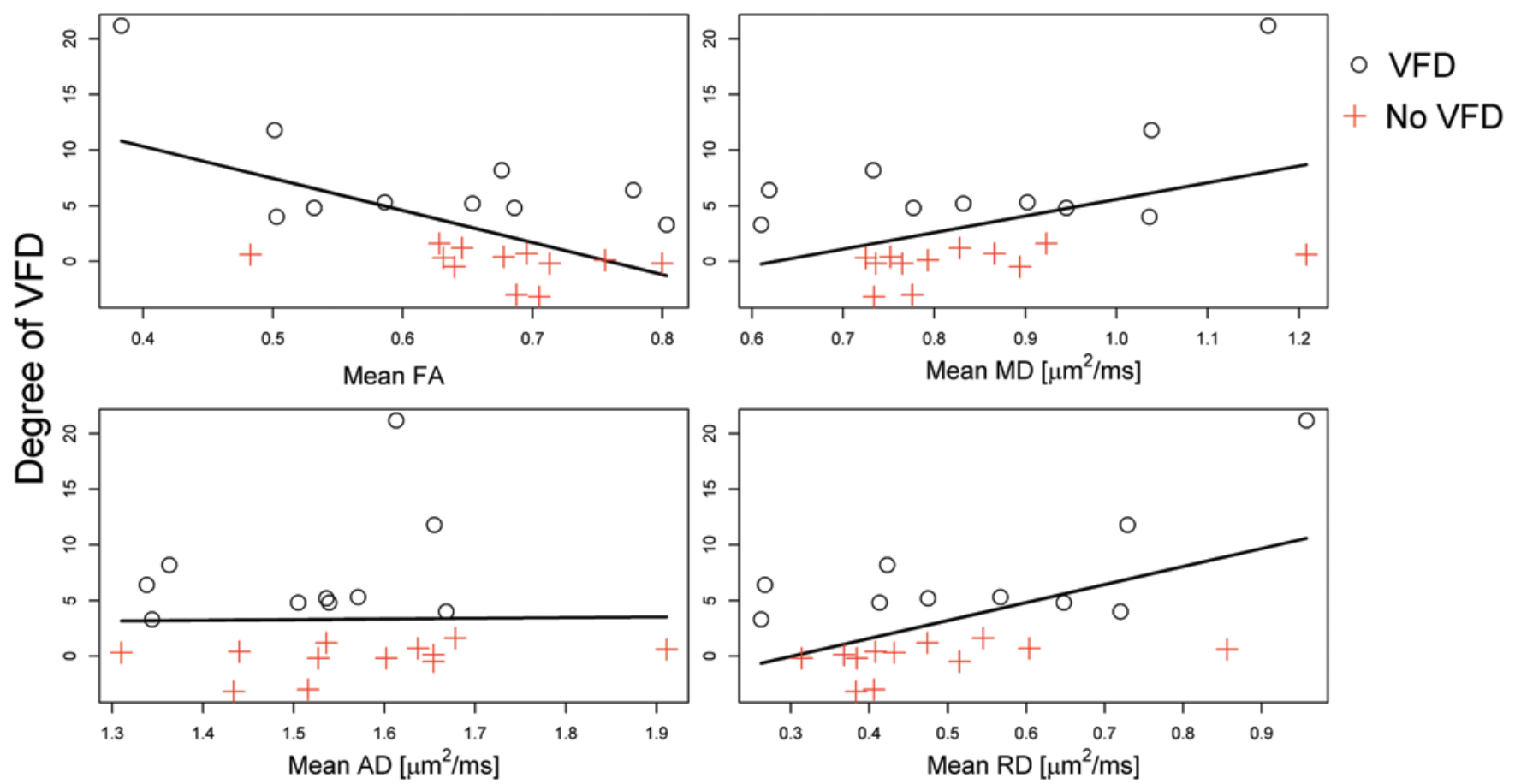

FIG. 4. Correlations between preoperative degree of VFD and DTI parameters (FA, MD, RD, and AD) from ROI 3. Statistical results are shown in Table 2. Figure is available in color online only.

chiasmal lift ( $r=-0.47, p<0.0 .5)$, but no significant correlations with $\mathrm{AD}$ (Fig. 4, Tables 2 and 3). The correlations reported in this paragraph correspond to those of ROI 3; similar correlations or trends toward correlations were also seen in ROI 2. There were no significant correlations with $\mathrm{AD}$ for VFD or chiasmal lift in any of the ROIs. In general, the correlations between DTI parameters and degree of VFD were stronger than those between DTI parameters and chiasmal lift.

The results revealed 2 suspected outliers: Case 18 (a patient with preoperative VFD) and Case 23 (a patient without preoperative VFD) (Fig. 4). Visual inspection of the scatter plots suggests that Case 18 is an extreme example regarding both chiasmal lift and degree of VFD, although following the direction of the slope based on the other patients. The results of Case 23, on the other hand, do not follow the slope and show a different relationship between DTI parameters and degree of VFD/chiasmal lift compared with the other patients. The reason is unknown; no obvious assessment errors could be found. Correlation analyses excluding 1 or both of the outliers were performed to test their effect on the results. Upon exclusion of Case 18, all significant correlations disappeared.

TABLE 2. Correlation: degree of VFD versus DTI parameters*

\begin{tabular}{ccc}
\hline DTI Parameter & ROI 2 & ROI 3 \\
\hline FA & $r=-0.48, p=0.024$ & $r=-0.58, p=0.0045$ \\
\hline MD & $r=0.43, p=0.046$ & $r=0.43, p=0.043$ \\
\hline AD & $r=0.21, p=0.35$ & $r=0.02, p=0.95$ \\
\hline RD & $r=0.47, p=0.026$ & $r=0.55, p=0.0081$ \\
\hline
\end{tabular}

* Pearson correlation coefficients $(r)$ and $p$ values are presented.
The exclusion of Case 23 led to stronger correlations and smaller $p$ values. The exclusion of both outliers produced results similar to the original ones (that is, with both cases included).

\section{Group Comparisons by Mixed Model}

There were significant differences in AD between controls and patients with VFD ( $\mathrm{p}<0.05)$, both before and after surgery (ROI 3). There were no significant differences in AD between the 2 patient groups (patients with and without VFD) or between the controls and patients without VFD. There were significant differences in FA between controls and patients with VFD before surgery (ROIs 2 and 3 ), but only a trend toward such a difference after surgery $(\mathrm{p}=0.058$, ROI 3$)$. Before surgery, the patients with VFD had lower FA than all other groups, although this difference was only significant compared with the controls. Also, before surgery, there was a trend toward higher RD in the patient group with VFD compared with the controls $(\mathrm{p}=0.073$, ROI 3) (Table 4).

Comparisons based on mean diffusivity (MD) showed no significant differences or trends toward differences be-

TABLE 3. Correlation: chiasmal lift versus DTI parameters*

\begin{tabular}{ccc}
\hline DTI Parameter & ROI 2 & ROI 3 \\
\hline FA & $r=-0.18, p=0.41$ & $r=-0.47, p=0.023$ \\
\hline MD & $r=0.08, p=0.72$ & $r=0.35, p=0.097$ \\
\hline$A D$ & $r=-0.14, p=0.53$ & $r=0.03, p=0.89$ \\
\hline RD & $r=0.14, p=0.54$ & $r=0.48, p=0.020$ \\
\hline
\end{tabular}

* Pearson correlation coefficients $(r)$ and $p$ values are presented. 


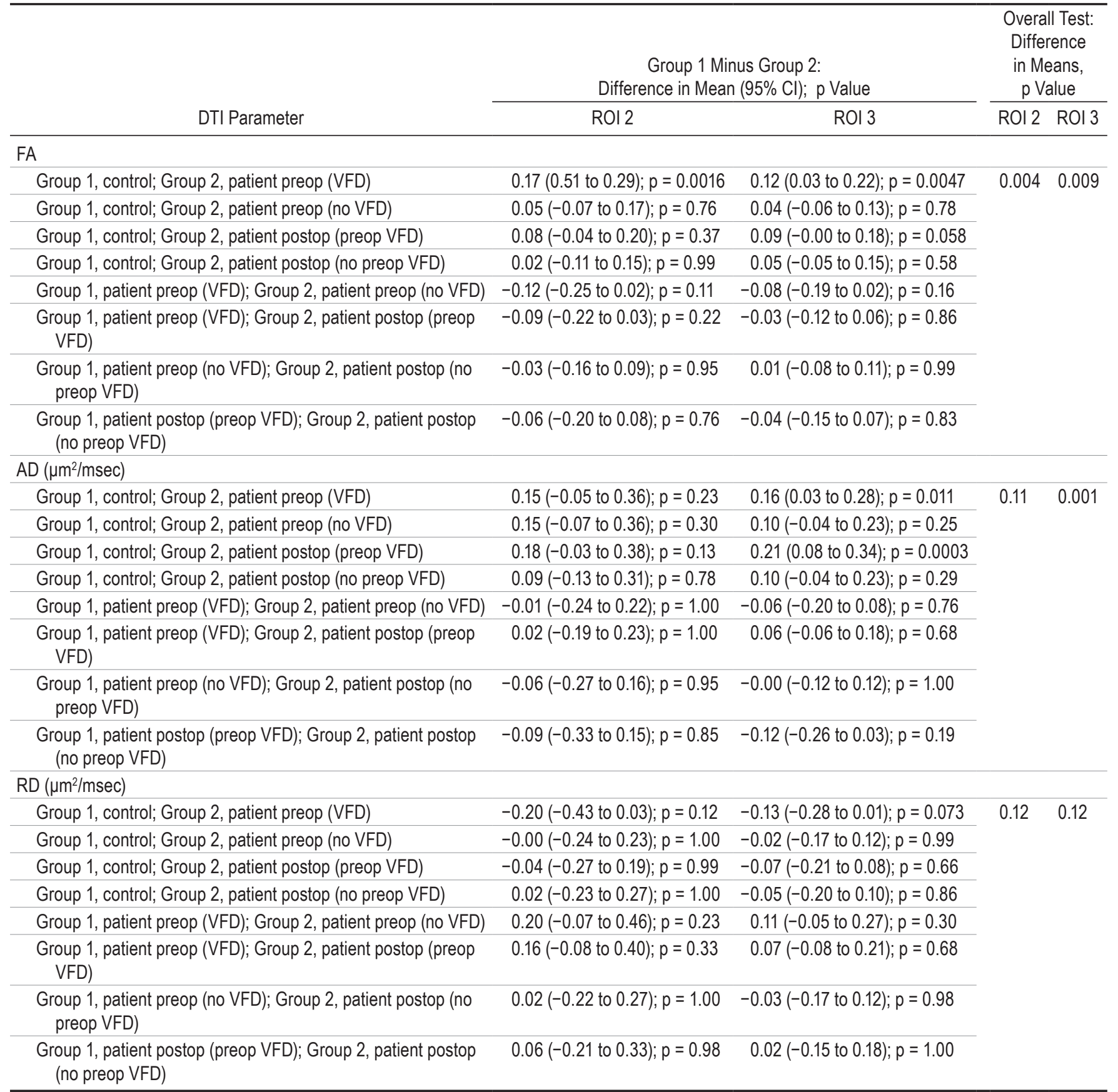

* Group comparisons are based on the mixed model analysis. The subjects were divided into 5 groups: 1) healthy control subjects, 2) patients with VFD before surgery (patient preop [VFD]), 3) patients without VFD before surgery (patient preop [no VFD]), 4) patients with preoperative VFD after surgery (patient postop [preop VFD]), and 5) patients without preoperative VFD after surgery (patient postop [no preop VFD]).

tween any of the groups ( $\mathrm{p}>0.5$ for all group comparisons). Results for MD are therefore not further presented.

\section{Prediction Model}

The selected prediction model was based on AD from ROIs 2 and 3 and FA from ROI 3. The maximum correlation between included predictors in this model was 0.14 . The model classified all patients with VFD correctly (sensitivity 1), whereas 17 of 20 controls were classified as controls (specificity 0.85 ). Nine of 12 patients without VFD were classified as patients (sensitivity 0.75 ). The robustness of the model, given by the cross validation, is presented in Fig. 5.

\section{Discussion}

In this study, DTI parameters from the OTs in patients 


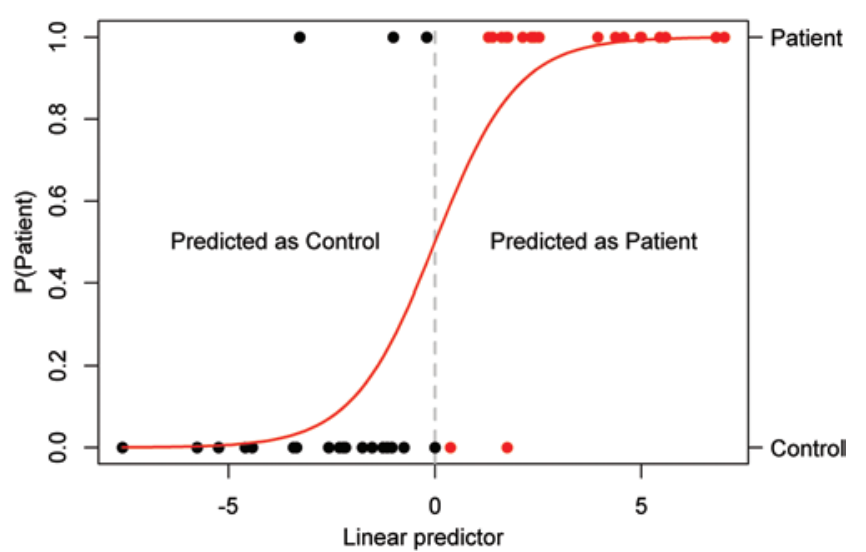

FIG. 5. Prediction model based on 3 predictors: $A D$ from ROIs 2 and 3 , and FA from ROI 3. Linear predictor: $50-16^{*} \mathrm{AD}_{\mathrm{RO} \mid 3}-22^{*} \mathrm{FA}_{\mathrm{ROI3}}-$ $6.1^{*} A D_{R O 12}\left(A D\right.$ in units of $\left.\mu m^{2} / m s e c\right)$. Area under the receiver operating characteristic curve: $0.91(95 \% \mathrm{Cl} 0.81-1.0)$. Figure is available in color online only.

with pituitary tumors were shown to correlate with the severity of VFD. These changes in diffusion parameters (FA and RD) may reflect levels of demyelination caused by the tumor compression. A prediction model, based on AD and FA alone, could separate patients from controls with high sensitivity. The greatest difference between patients with VFD and controls was seen in AD, which may represent a presence of axonal degeneration.

\section{VFD and Chiasmal Lift}

We found a strong correlation between the level of visual field impairment and chiasmal lift by the tumor (correlation coefficient $0.72, \mathrm{p}<0.05$ ). There are several possible measurement strategies for the quantification of both chiasmal lift and visual impairment. For this study, measurement methods resembling the ones defined by Frisén and Jensen were selected, which produced a correlation that was similar to theirs (Frisén and Jensen: $r=0.75, p<$ $0.05) .^{15}$

Schmalisch et al. measured the suprasellar extension of pituitary adenomas using a similar reference line, from the skull base, in search of objective data defining the risk of visual compromise. ${ }^{27}$ They found a correlation between visual-impairment score and suprasellar extension, and defined a suprasellar cut-off for the emergence of visual disturbance (87\% sensitivity, $72 \%-76 \%$ specificity).

Carrim et al. studied 19 patients with pituitary adenomas and quantified the level of chiasmal lift by measuring the distance between the chiasm and a reference line joining the 2 internal carotid arteries. ${ }^{6}$ They found a significant correlation between chiasmal lift and VFD $(\mathrm{r}=$ $0.55)$, based on degree of central bitemporal visual field loss. Wang et al. also found relationships between tumor height and VFD, although on the basis of fairly course classifications: 3 levels of suprasellar extension and 4 levels of VFD. ${ }^{33}$

It can be concluded that the suprasellar extension of the tumor alone can contribute to the objective assessment of visual impairment due to pituitary adenomas. However, the quantification methods suffer from inherent measurement errors, because the original position of the chiasm cannot be known once it has been affected by a tumor. Alternative anatomical landmarks have to be defined as reference points for measurement, and all such reference points have interindividual anatomical variabilities. In addition, other factors may play a role in the tumor effect on the anterior visual pathways, such as the duration of pressure and the exact point of pressure.

\section{Visual Pathway Injury Due to Compression by Tumor}

Several groups have studied the specific pathology caused by the external pressure by pituitary adenomas on the anterior visual pathways. Clinical follow-up studies of patients after tumor resection have reported at least 2 phases of visual recovery, which may correspond to the original mechanisms of injury: 1) an early phase that occurs within days after surgery, and 2) a later phase, within 1-4 months after surgery. ${ }^{18,21}$ A proposed explanation of the early recovery has been removal of conduction block and/or recovery of the cytoskeleton. The late recovery phase has been explained by remyelination, which is supported by studies in cats. Chronic compression of the cat optic nerve has been shown to lead to a gradual demyelination, in combination with partial axotomy. ${ }^{7,8}$

Cottee et al. also monitored nerve conduction after decompression, by means of implanted electrodes, and could observe a fast and late phase of recovery, concordant with the clinical studies. ${ }^{8}$ Microscopic analysis revealed a remyelination that followed the time course of the late recovery phase. There was no recovery of the axons that had undergone axotomy. Based on these reports, the variations of radial and axial diffusivities shown in this study may be caused by varying degrees of myelination and axonal degeneration, respectively, in the patient group. The intracellular compartment does not appear to be significant in the contribution to anisotropy; thus, possible intracellular causes for visual impairment would not affect the DTI parameters. ${ }^{4,5,32}$

\section{Prediction Model}

Several combinations of the 4 DTI parameters were assessed to construct a prediction model that could separate patients from controls. The most successful model was primarily based on AD in combination with FA (Fig. 5). According to the animal-model studies discussed herein, a decrease of AD may be a marker for axonal degeneration. ${ }^{3}$ The prediction model could thus reflect considerable differences in the level of axonal degeneration between the groups, in combination with more unspecific conditions affecting the anisotropy.

A certain level of axonal degeneration is likely in the group of patients with VFD, which could explain why the model classified all patients with VFD as patients. Local, less extensive axonal degeneration, in combination with demyelination, may be present in the group of patients without VFD, which may explain why 9 of the 12 patients without VFD could be classified as patients.

Kristof et al. studied visual outcome after resection of pituitary adenomas and observed that patients with a preoperative visual impairment improved postoperatively, but not to the level of patients with no preoperative visual impairment. ${ }^{22}$ Thus, surgery may be indicated before visual 
impairment develops to prevent irreversible injury. For such a purpose, methods that can identify early signs of injury are required. A prediction model like the one described herein may be such a method; however, it should be tested and confirmed in larger study groups. A model combining DTI parameters with chiasmal lift (another objective measure) may be an alternative.

\section{Axial Diffusivity}

The group comparisons revealed significantly lower axial diffusivities in the patient group with VFD compared with the control group, both before and after surgery (Table 4), consistent with the significance of AD in the prediction model. The irreversible nature of axonal degeneration would explain why these group differences were seen both before and 6 months after surgery. In contrast, there was no significant difference in AD between the control group and the patient group without VFD before surgery, which may be explained by an absence of axonal degeneration in this patient group. However, a difference in AD would then have been expected between patients with and without VFD before surgery, but no such difference was found. These seemingly contradictory findings may be due to the limited number of subjects in this study, which could conceal subtler group differences.

According to the neuroophthalmological assessment, only 3 of the 11 patients with VFD had optic nerve atrophy (before and after surgery), and only 4 of the patients with preoperative VFD had a postoperative VFD. However, the results of both the group comparisons and the prediction model suggest a more extensive presence of axonal atrophy in the OTs of the patients. It is likely that the first signs of injury occur in, or close to, the part of the anterior visual pathway that is in direct contact with the tumor, such as the OTs, which may be detected on DTI but not by conventional diagnostic methods.

\section{Radial Diffusivity}

In the present study, an increase of VFD was correlated with an increase in RD and a decrease in FA. This change in DTI parameters may be the result of a gradual demyelination, concurrent with a worsening of visual field impairment (Fig. 4, Table 2). ${ }^{3}$ The results are in accordance with previous work by Paul et al., who reported a positive correlation between RD and visual disability, based on an imaging study including 9 patients with compressive pituitary tumors. $^{24}$

The group comparisons herein revealed no significant differences in RD between any of the groups, although a trend could be observed between controls and patients with VFD before surgery. This trend disappeared after surgery, along with significant differences in FA before surgery between the same 2 groups. The difference in FA before surgery may be the result of a combination of affected axial and RD. The changes in RD and FA after decompressive surgery may be due to a remyelination and thus a normalization of the RD postoperatively.

Similarly, there were no significant differences in DTI parameters for the patient group with preoperative VFD before compared with after decompressive surgery, although all patients improved postoperatively with regard to visual field. However, although not statistically significant, some differences could be seen. After surgery, RD was lower and FA was higher, whereas there was almost no change in AD. Due to a limited study power, these results can neither confirm nor renounce a postoperative difference for patients with preoperative VFD. Reproduction of this study, with a larger patient cohort, would be of value.

In contrast to the results of the present study, Paul et al. reported significant differences in $\mathrm{RD}$, and no differences in $\mathrm{AD}$, in the OTs between 9 patients with compressive tumors and 9 controls. ${ }^{24}$ They also reported a normalization of RD after surgery for the patients with compressive tumors. The inconsistencies between the present study and the study by Paul et al. may be due to the fact that both studies were based on relatively few patients, who may have differed from each other in levels and types of OT injury. Furthermore, the present study has concentrated patients with more severe tumor impact in a single group (that is, patients with VFD); this may have concentrated patients with axonal atrophy in 1 group, which could be the reason for the detection of $\mathrm{AD}$ as a significant outcome variable. Also, the statistical methods used in the 2 studies differ; the machine-learning method used by Paul et al. may be more sensitive to small differences, but may also be more prone to overfitting.

\section{ROI Selection}

In most cases of pituitary adenomas, the optic chiasm is the first part of the visual pathway that is subject to pressure. However, in this study, the OTs were selected for analysis rather than the optic chiasm, due to the following reasons. The regular adult chiasm is a small structure (mean height $3 \mathrm{~mm}$, mean width $5 \mathrm{~mm}$ ) relative to regular DTI voxel sizes of approximately $2 \times 2 \times 2 \mathrm{~mm}^{3} .{ }^{23}$ The height of a compressed chiasm will often fall below the size of a voxel, and thus lead to increased partial volume effects in the voxels, as diffusivities from the surrounding CSF are included.

Anik et al. studied the effect of pituitary macroadenomas on the optic chiasm, in a retrospective DTI study including 72 patients. ${ }^{1}$ They reported a significant decrease in FA in the chiasm with increasing severity of VFD. However, according to the reasoning above, this decrease in FA may partly be due to increased partial volume effects in the ROI voxels from which data were extracted.

Furthermore, the standard DT model (that is, a secondorder tensor model) used by Anik et al., as well as in the present study, may be poorly suited for the optic chiasm. In the optic chiasm, there are several different fiber directions, as fibers corresponding to different parts of the visual fields are reorganized. As a consequence, the DTI parameters in such voxels will be an average of several fiber orientations. Thus, the second-order tensor model that assumes 1 fiber orientation per voxel may be poorly suited for the chiasm, including the defined ROI 1 herein, but well suited for the parallel organization of the OTs.

Previous work by our group, as well as a study by Paul et al., has revealed a pattern of varying DTI parameter values along the length of the OTs with, for example, lower FA close to the chiasm and an increase of FA more posteriorly. ${ }^{24}$ These changes in DTI parameters along the OT, 
as well as the inclusion of chiasmal voxels anteriorly, motivated the division into the 3 separate ROIs (ROIs 1,2, and 3 ). Based on the current results, an OT ROI covering a section of the OT corresponding to ROIs 2 and 3 in this study may be suitable for similar DTI analyses in the future.

There is no consensus on scan protocol or data analysis method for DTI, including DTI of the anterior visual pathways, although previous work has shown differences in results solely due to methodological variations. ${ }^{20} \mathrm{In}$ the present study, a magnetic field strength and scan protocol were selected with the aim to decrease influence of susceptibility artifacts while maintaining a high signal-to-noise ratio, with the potential to be performed and repeated in a clinical setting. The semiautomatic ROI model was carefully selected with the aim to correctly include the OTs and to avoid border-zone voxels, with as little measurement-dependent variability as possible.

\section{Limitations of the Study}

Results from the visual field assessment by HRP were used as a gold standard in the study, for both correlation tests and for the dichotomization into 2 patient groups, the latter in combination with clinical evaluation. The variability of perimetry results due to the subjectivity of patient performance has been discussed previously in this article. The subjectivity leads to a risk of misgrading and misclassification of patients, which may have affected the results of this study.

Each OT was divided into 3 ROIs based on number of voxels. However, the corresponding ROIs in each subject will not represent exactly the same part of the OT, due to interindividual anatomical differences such as lengths and thicknesses of the OTs.

The correlations showed that all patients except 1 (Case 23) followed specific patterns of DTI parameter values for their respective groups. There was no apparent reason for the deviating values of Case 23.

Preoperative optical coherence tomography of the retinal nerve fiber layer has been demonstrated to be predictive of postoperative visual outcome after decompressive surgery of pituitary adenomas ${ }^{9}$ and may thus provide useful preoperative information. However, optical coherence tomography was not included in the current study protocol because it was not available at our research facility at the start of this study.

\section{Conclusions}

DTI was able to detect pathology and degree of injury in the anterior visual pathways that were compressed by pituitary adenomas. Correlations were found between DTI parameters (FA and RD) and the severity of VFD. Our prediction model, based on AD and FA alone, could separate patients from controls with high sensitivity. DTI may provide objective data, detect early signs of injury, and be an additional diagnostic tool for determining indication for surgery in cases of pituitary adenomas. The results of this study should be confirmed in a larger patient cohort.

\section{Acknowledgments}

We are grateful for the expert statistical advice from Henrik
Imberg and his group at Statistiska Konsultgruppen. We also thank the Oxford Centre for Functional MRI of the Brain (FMRIB) Analysis Group for providing the FSL software. This study was supported by research grants from the Gothenburg Medical Society, the Göteborg Foundation for Neurological Research, the "De Blindas Vänner" foundation, the Swedish Medical Society, ALF GBG grant no. 11284, and Neuro-S Up ALF 77090 (agreement concerning research and education of doctors).

\section{References}

1. Anik I, Anik Y, Koc K, Ceylan S, Genc H, Altintas O, et al: Evaluation of early visual recovery in pituitary macroadenomas after endoscopic endonasal transphenoidal surgery: Quantitative assessment with diffusion tensor imaging (DTI). Acta Neurochir (Wien) 153:831-842, 2011

2. Basser PJ: Inferring microstructural features and the physiological state of tissues from diffusion-weighted images. NMR Biomed 8:333-344, 1995

3. Beaulieu C: The basis of anisotropic water diffusion in the nervous system - a technical review. NMR Biomed 15:435455, 2002

4. Beaulieu C, Allen PS: Determinants of anisotropic water diffusion in nerves. Magn Reson Med 31:394-400, 1994

5. Beaulieu C, Allen PS: Water diffusion in the giant axon of the squid: implications for diffusion-weighted MRI of the nervous system. Magn Reson Med 32:579-583, 1994

6. Carrim ZI, Reeks GA, Chohan AW, Dunn LT, Hadley DM: Predicting impairment of central vision from dimensions of the optic chiasm in patients with pituitary adenoma. Acta Neurochir (Wien) 149:255-260, 2007

7. Clifford-Jones RE, Landon DN, McDonald WI: Remyelination during optic nerve compression. J Neurol Sci 46:239243, 1980

8. Cottee LJ, Daniel C, Loh WS, Harrison BM, Burke W: Remyelination and recovery of conduction in cat optic nerve after demyelination by pressure. Exp Neurol 184:865-877, 2003

9. Danesh-Meyer HV, Wong A, Papchenko T, Matheos K, Stylli S, Nichols A, et al: Optical coherence tomography predicts visual outcome for pituitary tumors. J Clin Neurosci 22:1098-1104, 2015

10. Edal AL, Skjödt K, Nepper-Rasmussen HJ: SIPAP-a new MR classification for pituitary adenomas. Suprasellar, infrasellar, parasellar, anterior and posterior. Acta Radiol 38:30-36, 1997

11. Ezzat S, Asa SL, Couldwell WT, Barr CE, Dodge WE, Vance ML, et al: The prevalence of pituitary adenomas: a systematic review. Cancer 101:613-619, 2004

12. Foroozan R: Chiasmal syndromes. Curr Opin Ophthalmol 14:325-331, 2003

13. Frisén L: High-pass resolution perimetry. A clinical review. Doc Ophthalmol 83:1-25, 1993

14. Frisén L: High-pass resolution targets in peripheral vision. Ophthalmology 94:1104-1108, 1987

15. Frisén L, Jensen C: How robust is the optic chiasm? Perimetric and neuro-imaging correlations. Acta Neurol Scand 117:198-204, 2008

16. Gulani V, Webb AG, Duncan ID, Lauterbur PC: Apparent diffusion tensor measurements in myelin-deficient rat spinal cords. Magn Reson Med 45:191-195, 2001

17. Halvorsen H, Ramm-Pettersen J, Josefsen R, Rønning P, Reinlie S, Meling T, et al: Surgical complications after transsphenoidal microscopic and endoscopic surgery for pituitary adenoma: a consecutive series of 506 procedures. Acta Neurochir (Wien) 156:441-449, 2014

18. Jakobsson KE, Petruson B, Lindblom B: Dynamics of visual improvement following chiasmal decompression. Quantitative pre- and postoperative observations. Acta Ophthalmol Scand 80:512-516, 2002 
19. Jenkinson M, Bannister P, Brady M, Smith S: Improved optimization for the robust and accurate linear registration and motion correction of brain images. Neuroimage 17:825-841, 2002

20. Jones DK, Cercignani M: Twenty-five pitfalls in the analysis of diffusion MRI data. NMR Biomed 23:803-820, 2010

21. Kerrison JB, Lynn MJ, Baer CA, Newman SA, Biousse V, Newman NJ: Stages of improvement in visual fields after pituitary tumor resection. Am J Ophthalmol 130:813-820, 2000

22. Kristof RA, Kirchhofer D, Handzel D, Neuloh G, Schramm J, Mueller CA, et al: Pre-existing chiasma syndromes do not entirely remit following transsphenoidal surgery for pituitary adenomas. Acta Neurochir (Wien) 153:26-32, 2011

23. Parravano JG, Toledo A, Kucharczyk W: Dimensions of the optic nerves, chiasm, and tracts: MR quantitative comparison between patients with optic atrophy and normals. J Comput Assist Tomogr 17:688-690, 1993

24. Paul DA, Gaffin-Cahn E, Hintz EB, Adeclat GJ, Zhu T, Williams ZR, et al: White matter changes linked to visual recovery after nerve decompression. Sci Transl Med 6:266ra173, 2014

25. Petruson B, Jakobsson KE, Elfverson J, Bengtsson BA: Fiveyear follow-up of nonsecreting pituitary adenomas. Arch Otolaryngol Head Neck Surg 121:317-322, 1995

26. Powell M: Recovery of vision following transsphenoidal surgery for pituitary adenomas. Br J Neurosurg 9:367-373, 1995

27. Schmalisch K, Milian M, Schimitzek T, Lagrèze WA, Honegger J: Predictors for visual dysfunction in nonfunctioning pituitary adenomas-implications for neurosurgical management. Clin Endocrinol (Oxf) 77:728-734, 2012

28. Smith SM, Jenkinson M, Johansen-Berg H, Rueckert D, Nichols TE, Mackay CE, et al: Tract-based spatial statistics: voxelwise analysis of multi-subject diffusion data. Neuroimage 31:1487-1505, 2006

29. Song SK, Sun SW, Ju WK, Lin SJ, Cross AH, Neufeld AH: Diffusion tensor imaging detects and differentiates axon and myelin degeneration in mouse optic nerve after retinal ischemia. Neuroimage 20:1714-1722, 2003

30. Song SK, Yoshino J, Le TQ, Lin SJ, Sun SW, Cross AH, et al: Demyelination increases radial diffusivity in corpus callosum of mouse brain. Neuroimage 26:132-140, 2005

31. Tabaee A, Anand VK, Barrón Y, Hiltzik DH, Brown SM Kacker A, et al: Endoscopic pituitary surgery: a systematic review and meta-analysis. J Neurosurg 111:545-554, 2009

32. Takahashi M, Hackney DB, Zhang G, Wehrli SL, Wright AC, O’Brien WT, et al: Magnetic resonance microimaging of intraaxonal water diffusion in live excised lamprey spinal cord. Proc Natl Acad Sci U S A 99:16192-16196, 2002

33. Wang H, Sun W, Fu Z, Si Z, Zhu Y, Zhai G, et al: The pattern of visual impairment in patients with pituitary adenoma. $\mathbf{J}$ Int Med Res 36:1064-1069, 2008

34. Xu J, Sun SW, Naismith RT, Snyder AZ, Cross AH, Song SK: Assessing optic nerve pathology with diffusion MRI: from mouse to human. NMR Biomed 21:928-940, 2008

35. Yanoff M, Duker JS (eds): Ophthalmology, ed 3. Philadelphia: Mosby Elsevier, 2009

\section{Disclosures}

The authors report no conflict of interest concerning the materials or methods used in this study or the findings specified in this paper.

\section{Author Contributions}

Conception and design: Lilja, Ljungberg, Starck, Lindblom, Skoglund, Jakobsson, Nilsson. Acquisition of data: Lilja, Gustafsson, Ljungberg, Starck, Lindblom, Skoglund, Bergquist, Nilsson. Analysis and interpretation of data: Lilja, Gustafsson, Ljungberg, Starck, Nilsson. Drafting the article: Lilja, Gustafsson. Critically revising the article: all authors. Reviewed submitted version of manuscript: all authors. Approved the final version of the manuscript on behalf of all authors: Lilja. Statistical analysis: Lilja, Gustafsson. Study supervision: Ljungberg, Starck, Skoglund, Nilsson.

\section{Correspondence}

Ylva Lilja, Ear, Nose and Throat Clinic, Sahlgrenska University Hospital, Gröna stråket 5, Gothenburg 413 45, Sweden. email: ylva.lilja@neuro.gu.se. 